\title{
FIB-SEM Nanotomography in Materials and Life Science at EPFL
}

\author{
M. Cantoni, G. Knott and C. Hébert \\ EPFL-CIME, Ecole Polytechnique Féderale Lausanne, MXC-133, Station 12, 1015 Lausanne, \\ Switzerland
}

FIB-SEM microscopy at EPFL is applied in numerous disciplines of materials and life-science. Especially FIB-tomography is being used to understand complex microstructures in 3 dimensions. It combines FIB milling with the imaging capabilities and the variety of different detection modes (low $\mathrm{kV}$ BSE, EDX, EBSD, SIMS) of a modern SEM. We use FIB-tomography in areas were x-ray tomography does not offer the resolution and where TEM tilt-series tomography fails to cover the necessary volume to understand the microstructure. Through fully automated slicing and imaging we were able to generate stacks of up to 3300 images (slices) with rates of 40-70 images per hour. We use image pixel sizes of 5-10 nm and a corresponding slice thickness of 5-10 nm to generate stacks with isometric voxel sizes (3D pixel). These stacks can then ideally be used to reconstruct the volumes. With a voxel size of $5 \mathrm{~nm} \times 5 \mathrm{~nm} \times 5 \mathrm{~nm}$ and the accessible volumes of typically $20 \times 20 \times 20$ microns FIB tomography closes nicely the gap between X-ray tomography and TEM tilt series tomography.

\section{EXAMPLES OF FIB NANOTOMOGRAPHY}

The following examples show how versatile FIB nanotomography can be used to investigate the microstructure of nano-scaled materials.

Fig. 1 shows a small sub-volume of a $\mathrm{Nb}_{3} \mathrm{Sn}$ superconducting multifilament cable. The image contrast reveals not only the filament structure but also the grain size distribution within the filaments. The reconstructed volume in this case was 20x15x17 microns but only a sub-volume is display here.

In Fig. 2 the porous electrode of a solid-oxide fuel cell (SOFC) is shown. The different gray levels represent the metallic phase (white in the image, responsible for the electrical conductivity), the ceramic phase (ionic conductor) and the pores (gas feed). The volume covers in this case $24 \times 18 \times 18$ microns and permits the investigation of the connectivity between the different phases and the characterization of the triple points which play a key role for the perfect functionality of the device. In order to reduce milling artifacts the pores have been infiltrated with epoxy resin before the acquisition of the image stack.

Combining biological sample preparation techniques (fixation, substitution of water by epoxy resin and heavy metal staining) with FIB milling and SEM backscattered imaging gives a stunning insight into how cultured cells adhere on ceramic coated medical steel (implant). The ion beam mills trough biological tissue, epoxy resin, ceramic layers as well as the steel substrate in a similar way giving access to image contrasts that permit reconstructing the grain structure in the steel, the ceramics and also the internal structure of the cell (mitochondria, nucleus etc.), Fig.3.

We have also been exploring the use of FIB-SEM to visualize in three dimensions the ultrastructure of different brain regions. The aim has been to optimize the sample preparation and imaging parameters to produce the highest quality image series through volumes of tissue so that detailed analyses can be made of synaptic structure and brain connectivity (Fig.4). 
FIB applications and FIB Nanotomography have become indispensible tools in the electron microscopy center for the analysis of nanoscale structures in 3 dimensions.

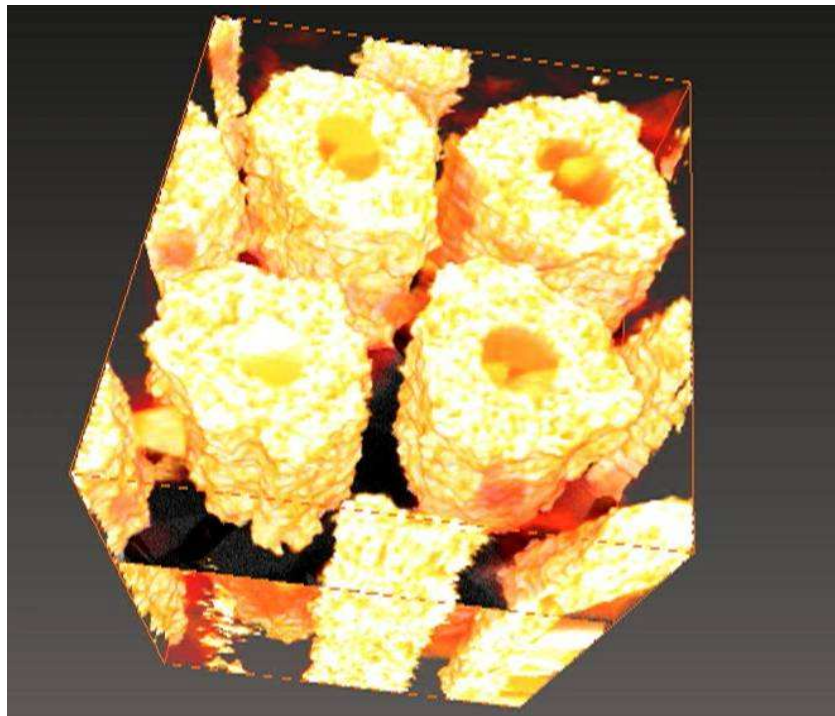

Fig. 1: $\mathrm{Nb}_{3} \mathrm{Sn}$ superconductor filaments: $2048 \times 1536$ pixels, 1700 slices $(10 \times 10 \times 10 \mathrm{~nm}$ voxel)

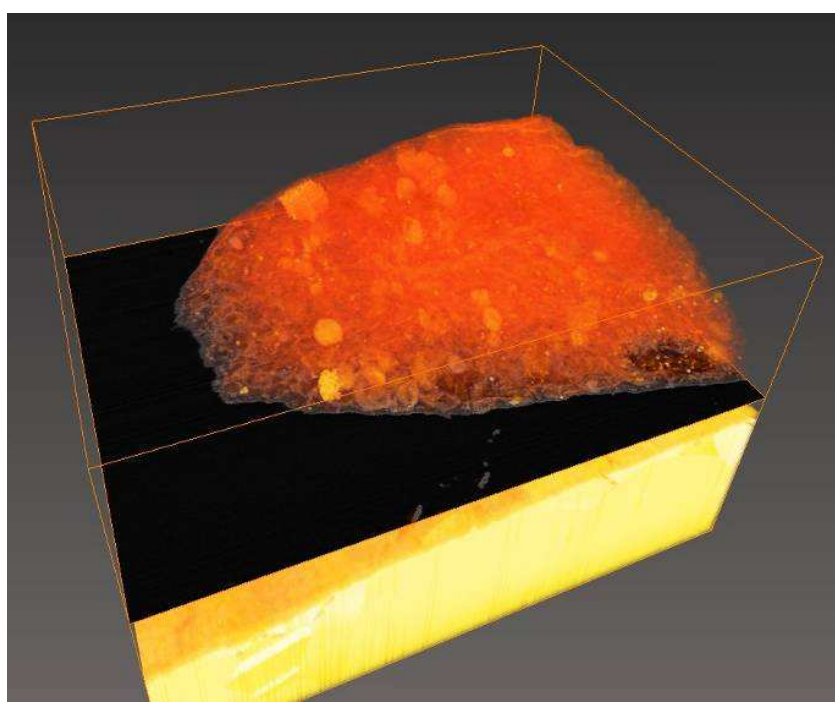

Fig. 3: Cell on substrate: 2048x1536 pixel, 600 slices (10x10x20nm voxel).

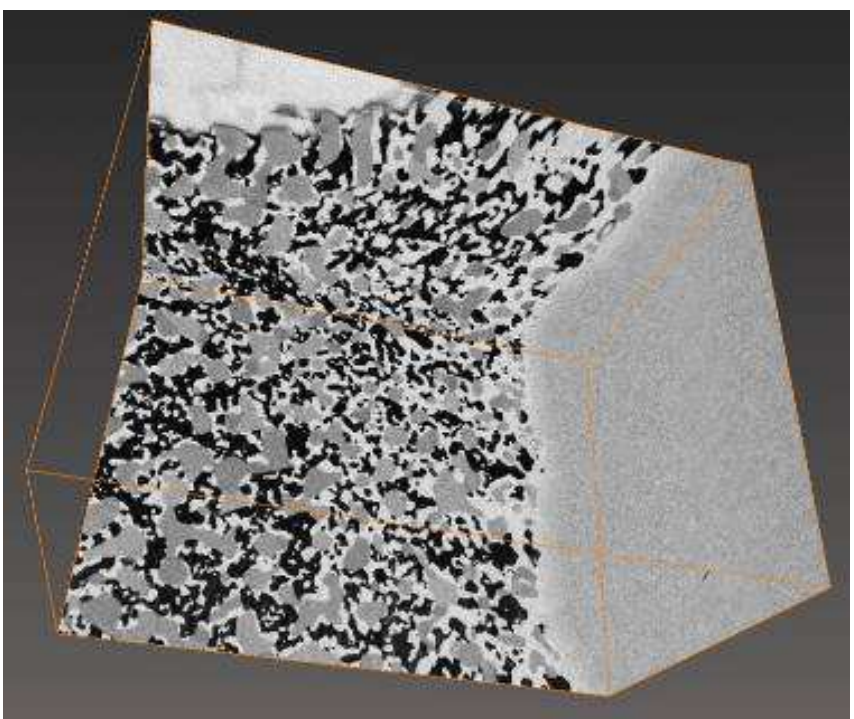

Fig. 2: SOFC electrode material, (porous Ni-CGO anode) 2048x1536 pixels, 1500 slices (12x12x12nm voxel)

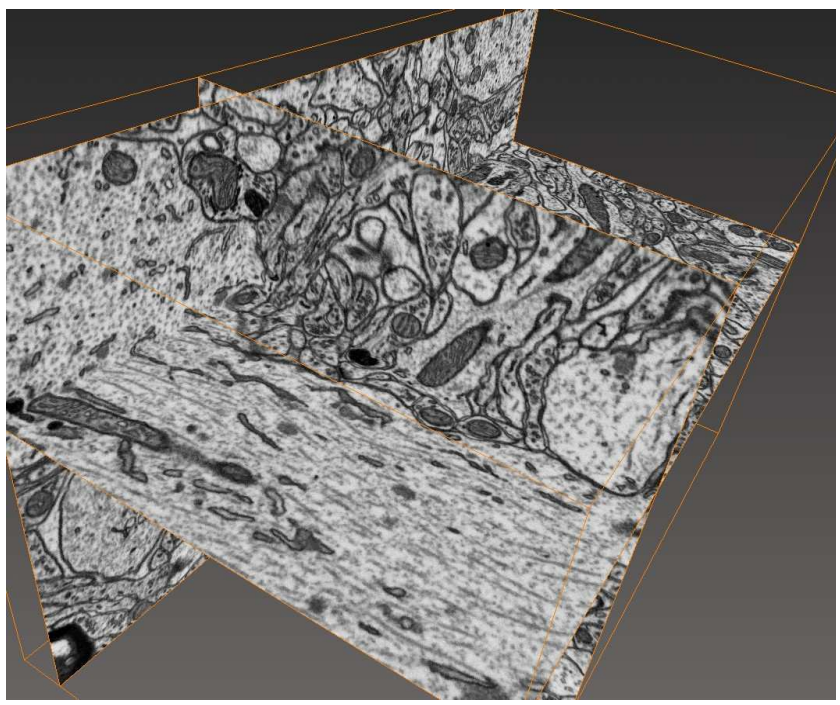

Fig. 4: mouse brain 3096x2306 pixels, 3300 slices $(7.5 \times 7.5 \times 7.5 \mathrm{~nm}$ voxel $)$ 